\title{
Naringin attenuates cerebral ischemia- reperfusion injury in rats by inhibiting endoplasmic reticulum stress
}

https://doi.org/10.1515/tnsci-2020-0170

received March 2, 2021; accepted April 16, 2021

\begin{abstract}
Objective - This investigation was carried out with an aim of exploring neuroprotection by naringin (Nar) in rats with cerebral ischemia-reperfusion $(\mathrm{CI} / \mathrm{R})$ injury and its mechanism.
\end{abstract}

Methods - Rats were grouped into ischemia-reperfusion $(\mathrm{I} / \mathrm{R})$, sham operation (Sham), nimodipine control (NIM), and different doses of Nar (Nar-L, Nar-M, Nar-H) groups. With Zea Longa score for assessment of neurological deficits, dry and wet method for measurement of brain tissue water content, and (2,3,5-triphenyltetrazolium chloride) TTC staining for determination of cerebral infarction volume, the related parameters were obtained and compared. Subsequently, ELISA was introduced to detect levels of proinflammatory cytokines (TNF- $\alpha$, IL-8) and anti-inflammatory cytokine (IL-10) in the serum as well as superoxide dismutase (SOD) and malondialdehyde (MDA) activities in brain tissue. Western blot was applied to evaluate endoplasmic reticulum stress (ERS)related proteins expression, including glucose-regulated protein 78 (GRP78), C/EBP homologous protein (CHOP), caspase-12, and activating transcription factor 6 (ATF-6). Results - Nar significantly alleviated nerve injury and decreased brain tissue water content and brain infraction volume in $\mathrm{CI} / \mathrm{R}$ injury rats in a concentration-dependent manner. Reduction of TNF- $\alpha$, IL-8 as well as MDA content

\footnotetext{
* Corresponding author: Haibin Wang, Department of Radiology, Translational Medicine Research Center, Key Laboratory of Clinical Cancer Pharmacology and Toxicology Research of Zhejiang Province, Affiliated Hangzhou First People's Hospital, Zhejiang University School of Medicine, Hangzhou 310006, China, e-mail: yue1988j@163.com

Li Wang: Department of Emergency Medicine, The Second Affiliated Hospital, Zhejiang University School of Medicine, Hangzhou 310002, China

Zhe Zhang: Department of Emergency Medicine, The First People's Hospital of Yuhang District, Hangzhou 311100, China
}

and elevation of IL-10 as well as SOD activity were confirmed to be caused by Nar treatment in a concentrationdependent manner. Meanwhile, ERS-related proteins also markedly decreased in the Nar groups.

Conclusion - Nar may achieve neuroprotection and alleviation of $\mathrm{CI} / \mathrm{R}$ injury by anti-inflammation, anti-oxidation, and inhibiting ERS, and its efficacy is concentrationdependent.

Keywords: naringin, cerebral ischemia-reperfusion injury, inflammation, oxidative stress, endoplasmic reticulum stress

\section{Introduction}

Cerebral ischemia-reperfusion $(\mathrm{CI} / \mathrm{R})$ injury, a cerebrovascular disease, occurs as a result of the restoration of blood perfusion after a certain period of cerebral ischemia and hypoxia, which will aggravate the structural and functional damage caused by hypoxia and even lead to more serious complications. $\mathrm{CI} / \mathrm{R}$ injurycaused severe brain injury and dysfunction is a challenging problem in clinical treatment, and currently no safe and reliable targeted drug was developed for it. Approximately 1 million CI/R injury-caused deaths and 5 million CI/R injury patients who become appear disability or paralysis has worldwide distribution [1]. With high incidence, disability, and mortality, CI/R injury seriously threatens health and life of patients and also leads to a marked reduction in their quality of life [2]. It has been shown that oxidative stress damage indirectly induced by $\mathrm{CI} / \mathrm{R}$ injury leads to physiological dysfunction of the endoplasmic reticulum, known as endoplasmic reticulum stress (ERS). ERS leads to endoplasmic reticulum protein misfolding, which further induces an unfolded protein response (UPR), while sustained oxidative stress and UPR can activate downstream apoptosis-related pathways and induce apoptosis [3]. And studies have also confirmed that the ERS-mediated apoptotic pathway is thought to play an important role in mediating brain 
damage after CI/R injury [4]. Therefore, how to effectively inhibit ERS induced by ischemia-reperfusion (I/R) injury will provide new ideas for the treatment of $\mathrm{CI} / \mathrm{R}$.

Many studies have found that natural extracts have a preventive effect on $\mathrm{CI} / \mathrm{R}$ injury [5]. For example, after being treated with sargentodoxa cuneata total phenolic acids, inflammation and oxidative response in brain tissue present to decline, thus relieving $\mathrm{CI} / \mathrm{R}$ injury in rats [6]; after being treated with total flavonoids of Radix Ilicis Pubescentis, damage of nerve cells in brain tissue and no content was appeared to be reduced, causing improvement in CI/R injury mice [7]. Naringin (Nar), a flavonoid extracted from the grapefruit fruit of the Rutaceae family, can act as free radical scavenger and is an effective antioxidation, anti-inflammation, and vascular protection. Many studies have shown that Nar has significant potential in the prevention and treatment of $\mathrm{CI} / \mathrm{R}$ injury [8]. Nar was found to reduce catalase activity, decrease peroxidation, and significantly improve neurological injury in $\mathrm{CI} / \mathrm{R}$ injury mice [9]. In addition, Nar could cross the blood-brain barrier and reduce excessive mitophagy, thus preventing $\mathrm{CI} / \mathrm{R}$ injury [10]. Wang et al. [11] found that Nar reduces oxidative stress and improves mitochondrial dysfunction by activating the Nrf2/ARE signaling pathway in neurons. Okuyama et al. [12] similarly demonstrated that Nar-rich rhizome peel has a neuroprotective effect against $\mathrm{CI} / \mathrm{R}$ injury in rats.

However, there are fewer studies related to the effect of Nar on ERS and the association with $\mathrm{CI} / \mathrm{R}$ injury. In view of the previous studies on Nar, we herein investigate the neuroprotection by Nar in $\mathrm{CI} / \mathrm{R}$ injury rats and the possible ERS-related mechanism of this extract.

\section{Materials and methods}

\subsection{Preparation of rat model of cerebral ischemia-reperfusion injury}

Fifty-four adult Sprague Dawley rats, 27 females and 27 males, weighing 180-220 g, were taken. Random allocation of the rats into 6 groups of 9 was completed, named as sham operation (Sham) group, ischemia/reperfusion (I/R) group, Nimodipine (NIM) group, and Nar low-, median-, and high-dosage (Nar-L, Nar-M, Nar-H) groups. For establishment of $\mathrm{I} / \mathrm{R}$ injury model, the rats were anesthetized with pentobarbital sodium $(40 \mathrm{mg} / \mathrm{kg}$, intraperitoneal injection), and endotracheal intubation was performed while spontaneous breathing was preserved.
After that, skin incision was followed to carefully expose the right common carotid artery, internal carotid artery, and external carotid artery. Then in the I/R group, the method described by Zea Longa was applied for blocking internal carotid artery to cause ischemia for $2 \mathrm{~h}$, followed by reperfusion for $24 \mathrm{~h}$. Intragastrical administration of normal saline $(1.8-2.2 \mathrm{~mL} / 100 \mathrm{~g}$, once a day) was given 7 days before surgery in the I/R group. In the Sham group, same preoperative treatment and same surgery as the $I / R$ group were done, but with no occlusion using nylon suture. In the NIM group, the surgery was also the same while intragastrical administration of the positive drug nimodipine $(15 \mathrm{mg} / \mathrm{kg})$ was given 7 days before surgery. The Nar groups received the same surgery, with intragastrical administration of 25, 50, and $100 \mathrm{mg} / \mathrm{kg}$ Nar 7 days before surgery.

\subsection{Neurological deficit score}

After $24 \mathrm{~h}$ of perfusion, Zea Longa score [13] was introduced for evaluation of neurological impairment in rats. 0 point: No nerve injury; 1 point: Failure to fully extend forepaw; 2 points: Contralateral circling; 3 points: Contralateral tumbling; 4 points: Failure to walk spontaneously and loss of consciousness.

\subsection{ELISA}

\subsubsection{Detection of inflammatory factors in serum}

On completion of neurological assessment, $12 \mathrm{~h}$ fasting was conducted with the provision of drinking. Next day, their blood was collected for $5 \mathrm{~min}$ centrifugation at $500 \mathrm{~g} / \mathrm{min}$ to take the serum. The final step was the assessment of TNF- $\alpha$, IL- 8 , and IL-10 contents in rat serum in keeping with the instructions of the ELISA kit (Abcam, UK) strictly.

\subsubsection{Detection of oxidative stress-related parameters in brain tissue}

After decapitation, brain tissues removed from 3 rats in each group were ground into homogenates for $10 \mathrm{~min}$ centrifugation at $500 \mathrm{~g} / \mathrm{min}$. Then the supernatant was taken. The final step was to measure superoxide dismutase (SOD) activity and malondialdehyde (MDA) content 
in keeping with the instruction of MDA kit and SOD kit (Nanjing Jiancheng Bioengineering Institute, China).

\subsection{Detection of brain tissue water content by a dry and wet method}

On completion of decapitation, brain tissues removed from 3 rats in each group were weighed to obtain the wet weight of the whole brain. The brain tissues were dried in a $105^{\circ} \mathrm{C}$ oven to a constant weight, which was the dry weight. Bran tissue water content $=$ (wet weight dry weight)/wet weight $\times 100 \%$.

\subsection{TTC staining}

On completion of decapitation, brain tissues removed from 3 rats in each group were cut into 5 coronal sections equidistantly. Then after $15 \mathrm{~min}$ incubation of these sections immersed in TTC solution, photographs were taken utilizing a digital camera and analysis of images was allowed by using ImageJ verl. 37c NIH software. Based on the determination of cerebral infarction area (pale area) and normal brain tissue area (red area), the percentage of cerebral infarction volume could be calculated.

\subsection{Western blot}

Brain tissues ground into homogenates was used to extract proteins by utilizing RIPA lysis buffer. Then in order to quantify extracted protein concentration, a BCA Kit (Thermo, USA) was applied. Then $25 \mu \mathrm{g}$ of protein was transported to the PVDF membrane after being separated utilizing SDS-PAGE. On completion of subsequent blocking step by $5 \%$ skim milk powder for a period of $2 \mathrm{~h}$, overnight co-incubation of the membrane and glucose-regulated protein 78 (GRP78), C/EBP homologous protein (CHOP), caspase-12, and activating transcription factor 6 (ATF-6) antibodies (Cell Signaling Technology, USA) was performed at $4^{\circ} \mathrm{C}$, followed by rinsing step for 3 times utilizing TBST and another $2 \mathrm{~h}$ incubation with corresponding secondary antibodies at room temperature. Once the incubation was completed, the sample was washed for 3 times again. Proteins were developed by a chemiluminescent reagent for a period of $3 \mathrm{~min}$. The final step was to obtain the images utilizing a gel imaging system and to analyze protein content utilizing QuantityOne software. Calculation of relative protein expression was allowed with GAPDH as an internal reference.

\subsection{Statistical analysis}

All data were presented as mean \pm standard deviation (SD). Statistical analysis was performed using SPSS 22.0. The differences between multiple groups were determined using one-way analysis of variance (ANOVA). Student's $t$-test analysis was performed to compare the difference between two groups. The criterion of a statistically significant difference was $P<0.05$.

Ethical approval: The research related to animals' use has been complied with all the relevant national regulations and institutional policies for the care and use of animals.

\section{Results}

\subsection{Remission of ischemia/reperfusion- induced brain injury by Nar}

In neurological impairment assessment, rats of the Sham group got 0 point. In comparison with the Sham group, the score representing brain injury severity increased markedly in the I/R group. In comparison with the I/R group, scores decreased markedly in the NIM group and Nar groups (Figure 1a). In the 3 Nar groups, with an escalation of Nar dosage, scores gradually decreased.

In further TTC staining and dry and wet methods, rats of Sham group showed no infarct and relatively low water content in the brain tissue. In comparison with the Sham group, different degrees of infarction and increases of water content could be observed in the other 5 groups (Figure $1 \mathrm{~b}$ ). In comparison with the $\mathrm{I} / \mathrm{R}$, nimodipine and Nar could significantly reduce the infarct size and brain water content of rat brain tissue, and Nar was concentration-dependent (Figure 1c and d). Hence, it was evident that Nar was effective in alleviating CI/R injuryinduced brain infarction and neurological impairment and in reducing brain tissue water content.

\subsection{Alleviation of ischemia/reperfusion- caused local inflammation by Nar}

From ELISA results, in comparison with the Sham group, serum TNF- $\alpha$ and IL-8 levels in the I/R group raised markedly, while IL-10 level decreased significantly. In comparison with the I/R group, serum TNF- $\alpha$ and IL-8 levels in the NIM group and Nar groups reduced considerably 
(a)

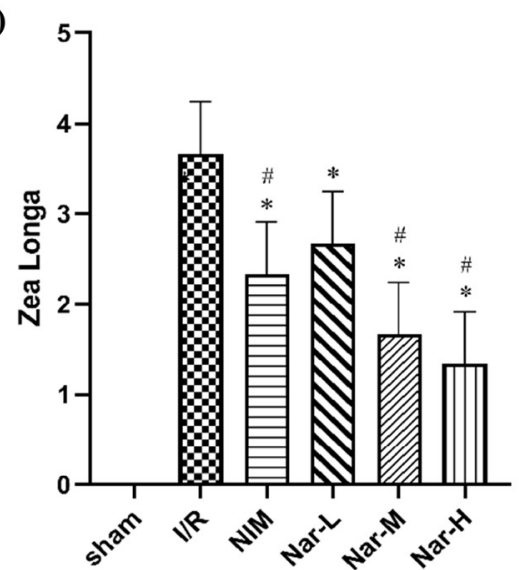

(c)

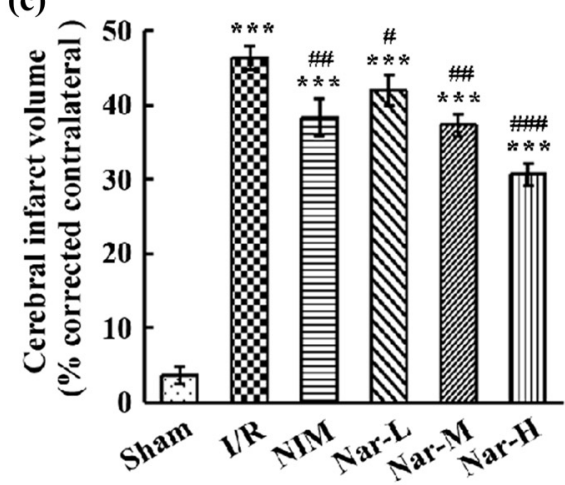

(b)

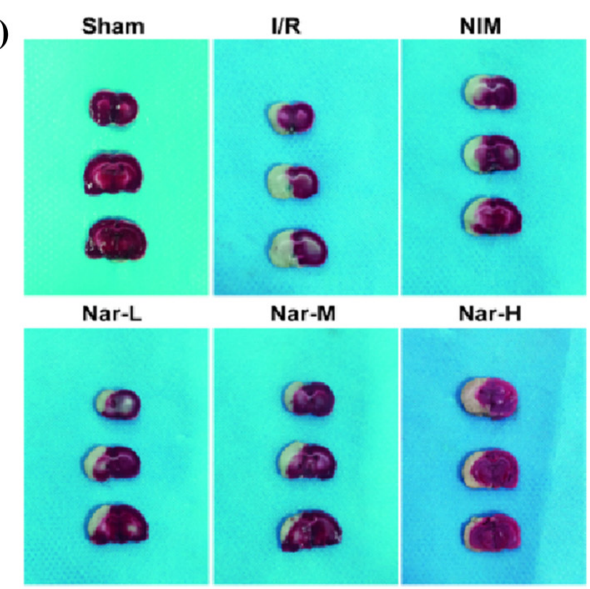

(d)

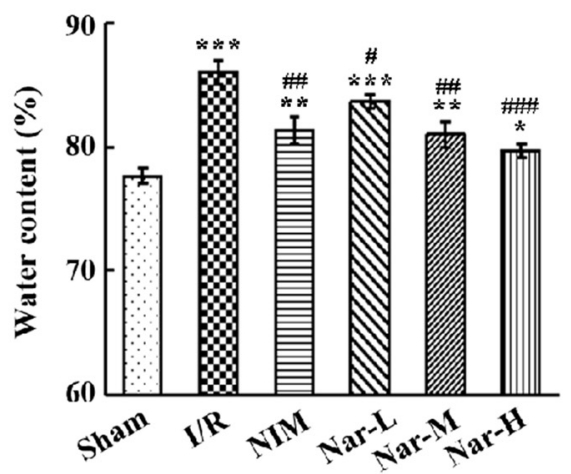

Figure 1: Remission of ischemia/reperfusion-induced brain injury by Nar. (a) Zea Longa score for neurological impairment assessment. (b) Determination of brain tissue infarction utilizing TTC staining. (c) Percentage of brain infarct volume. (d) Assessment of brain tissue water content utilizing dry and wet method. $N=3$ /group. ${ }^{\star} P<0.05,{ }^{\star \star} P<0.01$, and ${ }^{\star \star \star} P<0.001$ vs Sham group; ${ }^{\#} P<0.05$, ${ }^{\# \#} P<0.01$, and

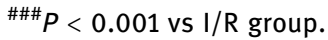

while IL-10 increased significantly, and Nar was concentration-dependent (Figure 2a-c). Hence, it was evident that Nar was effective in alleviating $\mathrm{CI} / \mathrm{R}$ injury-induced inflammation, and its efficacy was better than that of nimodipine when a certain concentration is reached.

\subsection{Inhibition of ischemia/reperfusion- caused oxidative stress by $\mathrm{Nar}$}

In comparison with the Sham group, I/R led to a marked reduction of SOD activity and an elevation of MDA content. In comparison with the I/R group, marked decrease of SOD activity and increase of MDA content were revealed in the NIM group and Nar groups (Figure 3a and b). And with the increase of Nar dose, SOD activity was on the rise and MDA content was on the decline, suggesting concentration-dependence of Nar. Compared with nimodipine, high dosage of Nar had better efficacy in reducing oxidative stress. Hence, it was supported that Nar, which functioned in increasing SOD activity and in decreasing MDA activity, could enhance ability to scavenge oxidative free radical, thus causing a reduction of peroxidation to relieve $\mathrm{CI} / \mathrm{R}$ injury-induced oxidative stress in rat brain tissue. Additionally, with the characteristic of concentration-dependence, Nar in a certain concentration could achieve better efficacy than nimodipine.

\subsection{Inhibition of ischemia/reperfusion- caused endoplasmic reticulum stress by Nar}

For the purpose of determination of the relationship between the neuroprotective effect of Nar and ERS, ERSrelated proteins in the brain tissues were assessed utilizing Western blot, including GRP78, CHOP, Caspase-12, and ATF6. I/R led to a marked increase of the expression of 
(a)

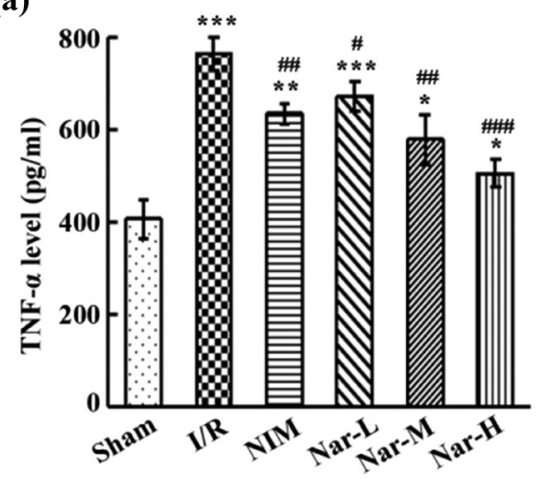

(b)

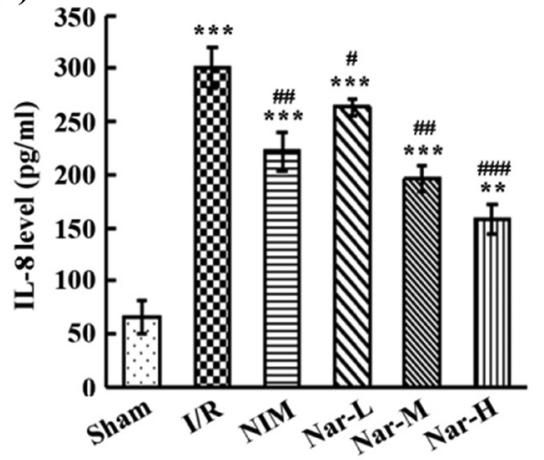

(c)

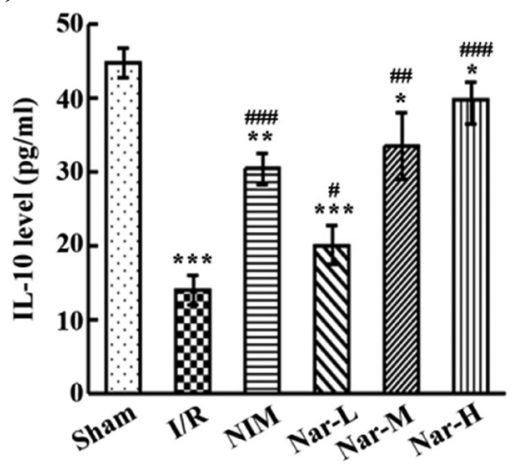

Figure 2: Alleviation of ischemia/reperfusion-caused inflammation by Nar. Evaluation of TNF- $\alpha$ (a), IL-8 (b), and IL-10 (c) levels in the serum of rats. $N=3$ /group. ${ }^{\star} P<0.05$, ${ }^{\star \star} P<0.01$, and ${ }^{\star \star \star} P<0.001$ vs Sham group; ${ }^{\#} P<0.05$, ${ }^{\# \#} P<0.01$, and ${ }^{\# \# \#} P<0.001$ vs $\mathrm{I} / \mathrm{R}$ group. This experiment was repeated three times.

these proteins, but nimodipine and Nar significantly decreased their expression (Figure 4). Nar was concentration-dependent. Collectively, inhibition of $\mathrm{CI} / \mathrm{R}$ injurycaused ERS by Nar was proved and this extract in a certain concentration could be more effective than nimodipine.

\section{Discussion}

After a period of cerebral ischemia, a significant functional and structural change will occur during blood flow recovery, which is known as $\mathrm{CI} / \mathrm{R}$ injury. This kind of blood flow recovery can lead to irreversible damage such as brain cell necrosis, cell swelling, and tissue with heterogeneous blood flow [14]. According to a large number of studies on $\mathrm{CI} / \mathrm{R}$ injury in recent years, $\mathrm{CI} / \mathrm{R}$ injury has been proved to be a key factor leading to brain injury, and the involvement of neuroinflammation and oxidative stress in the pathophysiological process of $C I / R$ injury has also been confirmed [15].

Based on a rat model of $I / R$ established by sutureoccluded method, we first explore the neuroprotection of Nar. The results showed that in comparison with the I/R group, administration of Nar led to higher nerve injury score, decreased infraction volume, and reduced water content in brain tissue, and its efficacy was concentration-dependent. Collectively, the improvement of $\mathrm{CI} / \mathrm{R}$ injury-caused nerve injury and brain tissue injury by Nar is supported by these outcomes. Previous studies have performed trials which determine that cerebral ischemia is a cause of injury in neuronal cell membranes and glial cells, further resulting in a significant increase in the levels of proinflammatory cytokines TNF- $\alpha$ and IL-8, and their increased levels are related to brain injury severity [16]. Both alleviation of $\mathrm{CI} / \mathrm{R}$ injury and
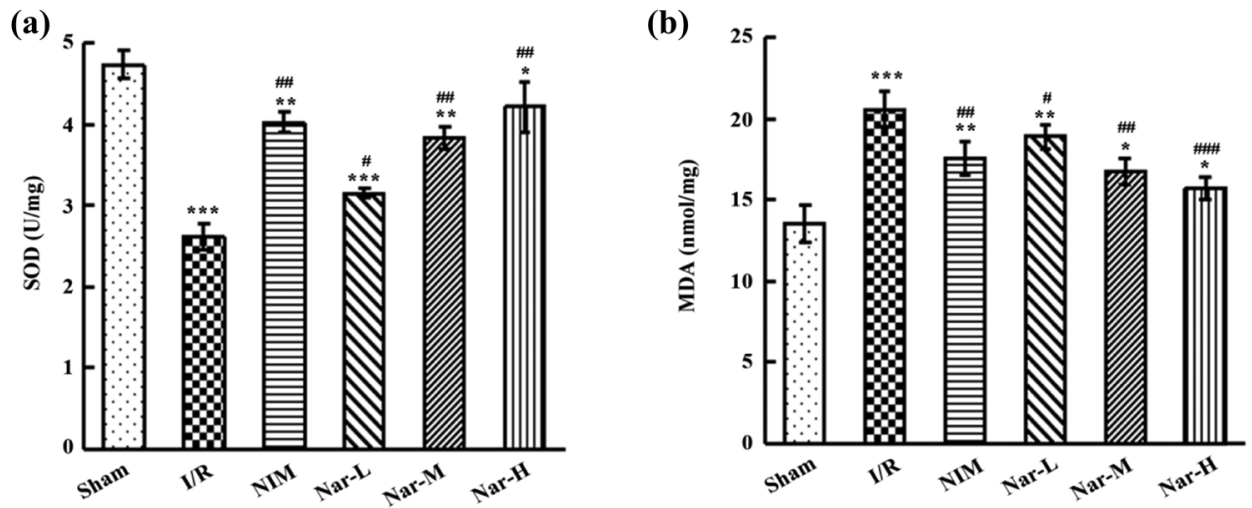

Figure 3: Inhibition of ischemia/reperfusion-caused oxidative stress by Nar. (a) Determination of SOD activity in brain tissue utilizing SOD kit. (b) Assessment of MDA content in brain tissue utilizing MDA kit. $N=3$ /group. ${ }^{\star} P<0.05,{ }^{*} P<0.01$, and ${ }^{\star \star \star} P<0.001$ vs Sham group; ${ }^{\#} P<0.05$, ${ }^{\# \#} P<0.01$, and ${ }^{\# \# \#} P<0.001$ vs $I / R$ group. This experiment was repeated three times. 


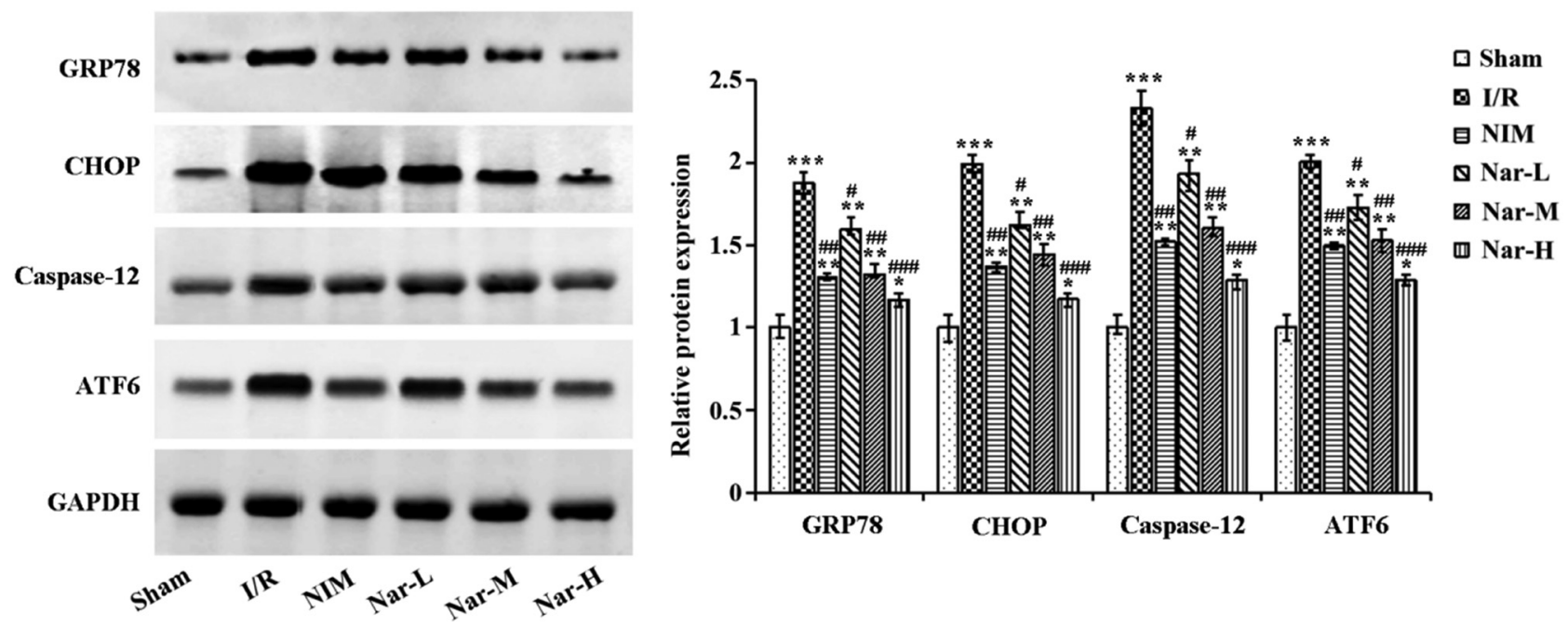

Figure 4: Inhibition of ischemia/reperfusion-caused endoplasmic reticulum stress by Nar. Determination of endoplasmic reticulum stressrelated proteins GRP78, CHOP, caspase-12, and ATF6 in the brain tissues utilizing western blot. $N=3 /$ group. ${ }^{\star} P<0.05$, $* \star P<0.01$, and ${ }^{\star \star \star} P<0.001$ vs Sham group; ${ }^{\#}<0.05,{ }^{\# \#} P<0.01$, and ${ }^{\# \# \#} P<0.001$ vs I/R group. This experiment was repeated three times.

improvement of stroke prognosis by anti-inflammatory regents and antioxidants have been proved $[17,18]$. Bai et al. pointed out the reduction of neuroinflammation and enhancement of neurological rehabilitation by IL-10 in rats with spinal cord injury. However, IL-10 level was appeared to be decreased when TNF- $\alpha$, IL- 8 , and IL- 6 levels increased accompanied by $24 \mathrm{~h}$ of middle cerebral artery occlusion [6]. Confirmed by Miao et al. oxidative stress after cerebral ischemia aggravated brain injury, while upregulation of SOD and catalase, which have antioxidant effects, facilitated the recovery of neurons underwent I/R [19]. Previous studies have shown that Nar has a strong antioxidant capacity and the ability to scavenge hydroxyl and superoxide radicals [20,21]. Sugumar et al. also found neuroprotective effects of naringenin against MPTP-induced oxidative stress [22]. For the resolution of the question of how Nar affects CI/R injury-caused inflammation and oxidative stress, ELISA was carried out in our study and found Nar could dose-dependently downregulate TNF- $\alpha$ and IL- 8 and MDA, while upregulate IL-10 and SOD. This result means that Nar can enhance ability to inhibit $\mathrm{CI} / \mathrm{R}$ injury-caused inflammation and to scavenge oxidative free radical, thus causing a reduction of peroxidation to relieve $\mathrm{CI} / \mathrm{R}$ injury-induced oxidative stress, which is as the previous studies. Collectively, it is speculated that anti-inflammation and anti-oxidative stress of Nar is effective in alleviating neuronal apoptosis, in inhibiting reactive glial cell activation and proliferation, and in improving ischemia-caused neurological impairment.
$\mathrm{CI} / \mathrm{R}$ injury leads to cell dysfunction, injury or death, and subsequent calcium overload, which triggers subsequent oxidative stress, organelle dysfunction, metabolic disorders as well as inflammation. Further, ischemia, hypoxia, and oxidative stress can induce ERS [23]. And many studies have shown that early administration of natural plant extracts improves CI/R injury. For example, suppression of ERS-mediated neuronal apoptosis by Iridoid glycosides from Radix Scrophulariae was proved, thus reducing local $\mathrm{CI} / \mathrm{R}$ injury in rats [24]. And downregulation of NF- $\mathrm{\kappa B}$ signaling pathway by celastrol was confirmed to cause neuroprotection in ischemic rats [25]. For further clarification of the relationship between the neuroprotection by Nar and ERS, measurement of ERSrelated proteins GRP78, CHOP, caspase-12, and ATF6 by western blot was conducted. The results showed that in comparison with the I/R group, Nar group presented a significant reduction of their expression. Among these proteins, ATF6 pathway is activated after ERS [26]. GRP78, a member of the heat shock protein 70 (HSP70) family, is mainly distributed in the ER [27]. Normally, GRP78 binds to the transmembrane proteins PERK, IRE1, and ATF6 and is unable to activate downstream signaling pathways. However, once the UPR is triggered, GRP78 will dissociate from PERK, ATF-6, and IRE1 and bind to the misfolded protein [28]. This in turn induces changes in the ER-mediated apoptotic pathway proteins caspase-12 and CHOP, ultimately inducing apoptosis [29,30]. And several studies have shown that apoptosis advances the pathological process of CIRI [31-33]. Tang et al. [34] also 
demonstrated that Nar inhibited hypoxia/reoxygenationinduced ATF6 expression in $\mathrm{H} 9 \mathrm{C} 2$ cells, which in turn inhibited ERS-mediated apoptosis. Thus, in the present study, we hypothesized that Nar could improve CI/R injury by inhibiting ERS.

\section{Conclusion}

Nar may achieve neuroprotection and alleviation of $\mathrm{CI} / \mathrm{R}$ injury by anti-inflammation, anti-oxidation, and inhibiting ERS, and its efficacy is better than nimodipine after reaching a certain concentration. However, due to the diversity in the efficacy of Nar, its specific molecular mechanism in inhibiting ERS and improving $\mathrm{CI} / \mathrm{R}$ injury still needs to be verified by subsequent studies, thus ensuring its safety and efficacy in clinical prevention and treatment of $\mathrm{CI} / \mathrm{R}$ injury.

Funding information: Hangzhou Science and Technology Development Plan Project (20191203B90).

Conflict of interest: Authors state no conflict of interest

Data availability statement: The datasets generated during and/or analyzed during the current study are available from the corresponding author on reasonable request.

\section{References}

[1] Soares RO, Losada DM, Jordani MC, Évora P, Castro-E-Silva O. Ischemia/reperfusion injury revisited: an overview of the latest pharmacological strategies. Int J Mol Sci. 2019 Oct;20(20):5034.

[2] Tian F, Liu R, Fan C, Sun Y, Huang X, Nie Z, et al. Effects of thymoquinone on small-molecule metabolites in a rat model of cerebral ischemia reperfusion injury assessed using MALDIMSI. Metabolites. 2020 Jan;10(1):27.

[3] Cnop M, Toivonen S, Igoillo-Esteve M, Salpea P. Endoplasmic reticulum stress and elF2 $\alpha$ phosphorylation: the Achilles heel of pancreatic $\beta$ cells. Mol Metab.

2017 Jul;6(9):1024-39.

[4] Feng SQ, Zong SY, Liu JX, Chen Y, Xu R, Yin X, et al. VEGF antagonism attenuates cerebral ischemia/reperfusioninduced injury via inhibiting endoplasmic reticulum stressmediated apoptosis. Biol Pharm Bull. 2019 May;42(5):692-702.

[5] Wu PF, Zhang Z, Wang F, Chen JG. Natural compounds from traditional medicinal herbs in the treatment of cerebral ischemia/reperfusion injury. Acta Pharmacol Sin. 2010 Dec;31(12):1523-31.
[6] Bai M, Liu B, Peng M, Jia J, Fang X, Miao M. Effect of Sargentodoxa cuneata total phenolic acids on focal cerebral ischemia reperfusion injury rats model. Saudi J Biol Sci. 2019 Mar;26(3):569-76.

[7] Yan X, Li H, Bai M, Miao M. Effect of total flavonoids of Radix Ilicis pubescentis on cerebral ischemia reperfusion model. Saudi J Biol Sci. 2017 Mar;24(3):595-602.

[8] Yang J, Yuan L, Wen Y, Zhou H, Jiang W, Xu D, et al. Protective effects of naringin in cerebral infarction and its molecular mechanism. Med Sci Monit. 2020 Jan;26:e918772.

[9] Gaur V, Aggarwal A, Kumar A. Protective effect of naringin against ischemic reperfusion cerebral injury: possible neurobehavioral, biochemical and cellular alterations in rat brain. Eur J Pharmacol. 2009 Aug;616(1-3):147-54.

[10] Feng J, Chen X, Lu S, Li W, Yang D, Su W, et al. Naringin attenuates cerebral ischemia-reperfusion injury through inhibiting peroxynitrite-mediated mitophagy activation. Mol Neurobiol. 2018 Dec;55(12):9029-42.

[11] Wang K, Chen Z, Huang L, Meng B, Zhou X, Wen X, et al. Naringenin reduces oxidative stress and improves mitochondrial dysfunction via activation of the Nrf2/ARE signaling pathway in neurons. Int J Mol Med. 2017 Nov;40(5):1582-90.

[12] Okuyama S, Yamamoto K, Mori H, Sawamoto A, Amakura Y, Yoshimura M, et al. Neuroprotective effect of Citrus kawachiensis (Kawachi Bankan) peels, a rich source of naringin, against global cerebral ischemia/reperfusion injury in mice. Biosci Biotechnol Biochem. 2018 Jul;82(7):1216-24.

[13] Longa EZ, Weinstein PR, Carlson S, Cummins R. Reversible middle cerebral artery occlusion without craniectomy in rats. Stroke. 1989 Jan;20(1):84-91.

[14] Shvedova M, Anfinogenova Y, Atochina-Vasserman EN, Schepetkin IA, Atochin DN. c-Jun N-terminal kinases (JNKs) in myocardial and cerebral ischemia/reperfusion injury. Front Pharmacol. 2018 Jul;9:715.

[15] Chen C, Qin H, Tan J, Hu Z, Zeng L. The role of ubiquitin-proteasome pathway and autophagy-lysosome pathway in cerebral ischemia. Oxid Med Cell Longev. 2020 Jan;2020:5457049.

[16] Janyou A, Wicha P, Jittiwat J, Suksamrarn A, Tocharus C, Tocharus J. Dihydrocapsaicin attenuates blood brain barrier and cerebral damage in focal cerebral ischemia/reperfusion via oxidative stress and inflammatory. Sci Rep. 2017 Sep;7(1):10556.

[17] Peng T, Jiang Y, Farhan M, Lazarovici P, Chen L, Zheng W. Antiinflammatory effects of traditional chinese medicines on preclinical in vivo models of brain ischemia-reperfusion-injury: prospects for neuroprotective drug discovery and therapy. Front Pharmacol. 2019 Mar;10:204.

[18] Kim IH, Lee TK, Cho JH, Lee JC, Park JH, Ahn JH, et al. Pretreatment with Chrysanthemum indicum Linné extract protects pyramidal neurons from transient cerebral ischemia via increasing antioxidants in the gerbil hippocampal CA1 region. Mol Med Rep. 2017 Jul;16(1):133-42.

[19] Miao M, Yan X, Guo L, Shao S. Effects of the Rabdosia rubescens total flavonoids on focal cerebral ischemia reperfusion model in rats. Saudi Pharm J. 2017 May;25(4):607-14.

[20] Nouri Z, Fakhri S, El-Senduny FF, Sanadgol N, Abd-ElGhani GE, Farzaei $\mathrm{MH}$, et al. On the neuroprotective effects of naringenin: pharmacological targets, signaling pathways, molecular mechanisms, and clinical perspective. Biomolecules. 2019 Nov;9(11):690. 
[21] Cavia-Saiz M, Busto MD, Pilar-Izquierdo MC, Ortega N, PerezMateos M, Muñiz P. Antioxidant properties, radical scavenging activity and biomolecule protection capacity of flavonoid naringenin and its glycoside naringin: a comparative study. J Sci Food Agric. 2010 May;90(7):1238-44.

[22] Sugumar M, Sevanan M, Sekar S. Neuroprotective effect of naringenin against MPTP-induced oxidative stress. Int J Neurosci. 2019 Jun;129(6):534-9.

[23] Chong WC, Shastri MD, Eri R. Endoplasmic reticulum stress and oxidative stress: a vicious nexus implicated in bowel disease pathophysiology. Int J Mol Sci. 2017 Apr;18(4):E771.

[24] Chen Y, Zhang L, Gong X, Gong H, Cheng R, Qiu F, et al. Iridoid glycosides from Radix Scrophulariae attenuates focal cerebral ischemia-reperfusion injury via inhibiting endoplasmic reticulum stress-mediated neuronal apoptosis in rats. Mol Med Rep. 2020 Jan;21(1):131-40.

[25] Zhang B, Zhong Q, Chen X, Wu X, Sha R, Song G, et al. Neuroprotective effects of celastrol on transient global cerebral ischemia rats via regulating $\mathrm{HMGB} 1 / \mathrm{NF}-\mathrm{KB}$ signaling pathway. Front Neurosci. 2020 Aug;14:847.

[26] Ron D, Walter P. Signal integration in the endoplasmic reticulum unfolded protein response. Nat Rev Mol Cell Biol. 2007 Jul;8(7):519-29.

[27] Li Y, Yi M, Wang D, Zhang Q, Yang L, Yang C. LncRNA KCNQ10T1 regulates endoplasmic reticulum stress to affect cerebral ischemia-reperfusion injury through targeting miR-30b/ GRP78. Inflammation. 2020 Dec;43(6):2264-75.

[28] Cook KL, Soto-Pantoja DR, Clarke PA, Cruz MI, Zwart A, Wärri A, et al. Endoplasmic reticulum stress protein GRP78 modulates lipid metabolism to control drug sensitivity and antitumor immunity in breast cancer. Cancer Res. 2016

Oct;76(19):5657-70.

[29] Zhang GL, Wang LH, Liu XY, Zhang YX, Hu MY, Liu L, et al. Cerebral dopamine neurotrophic factor (CDNF) has neuroprotective effects against cerebral ischemia that may occur through the endoplasmic reticulum stress pathway. Int J Mol Sci. 2018 Jun;19(7):1905.

[30] Ghosh AP, Klocke BJ, Ballestas ME, Roth KA. CHOP potentially co-operates with FOXO3a in neuronal cells to regulate PUMA and BIM expression in response to ER stress. PLoS One. 2012;7(6):e39586.

[31] Kishimoto M, Suenaga J, Takase H, Araki K, Yao T, Fujimura T, et al. Oxidative stress-responsive apoptosis inducing protein (ORAIP) plays a critical role in cerebral ischemia/reperfusion injury. Sci Rep. 2019 Sep;9(1):13512.

[32] Chen X, Cheng C, Zuo X, Huang W. Astragalin alleviates cerebral ischemia-reperfusion injury by improving anti-oxidant and anti-inflammatory activities and inhibiting apoptosis pathway in rats. BMC Complement Med Ther. 2020 Apr;20(1):120.

[33] Liu J, Wang Q, Yang S, Huang J, Feng X, Peng J, et al. Electroacupuncture inhibits apoptosis of peri-ischemic regions via modulating $\mathrm{p} 38$, extracellular signal-regulated kinase (ERK1/2), and c-Jun $\mathrm{N}$ terminal kinases (JNK) in cerebral ischemia-reperfusion-injured rats. Med Sci Monit. 2018 Jun;24:4395-404.

[34] Tang JY, Jin P, He Q, Lu LH, Ma JP, Gao WL, et al. Naringenin ameliorates hypoxia/reoxygenation-induced endoplasmic reticulum stress-mediated apoptosis in $\mathrm{H} 9 \mathrm{c} 2$ myocardial cells: involvement in ATF6, IRE1 $\alpha$ and PERK signaling activation. Mol Cell Biochem. 2017 Jan;424(1-2):111-22. 